\title{
Enunciación
}

http://revistas.udistrital.edu.co/ojs/index.php/enunc

DOI: http://dx.doi.org/10.14483/udistrital.jour.enunc.2015.2.a01

enunciación

Artículo de investigación

\section{Formar profesores de lenguaje y comunicación en y para la diversidad: una propuesta desde Alter-Nativa}

\section{Language and communication teachers' professional development in and for diversity: An Alter-Native proposal}

\author{
Dora Inés Calderónn ${ }^{1}$, Sandra Soler Castillo²
}

Para citar este artículo: Calderón, D. y Soler, S. (2015). Formar profesores de lenguaje y comunicación en y para la diversidad: una propuesta desde Alter-Nativa. Enunciación, 20(2), pp.174-189.

Recibido: 30-septiembre-2015 / Aprobado: 01-noviembre-2015

Cuántas veces aún soñar con un lenguaje no sometido a las palabras...

M. Blanchot

\section{Resumen}

En este escrito se presentan los resultados de una investigación pedagógica, de tipo curricular y didáctico, realizada en el marco de la acción Alter-Nativa, Alfa III. Específicamente, se exponen los resultados del trabajo mancomunado de la comunidad de formadores de profesores del área de lenguaje de América Latina y el Caribe, que conformaron el equipo de lenguaje Alter-Nativa. Esta comunidad propuso lineamientos comunes para la formación de profesores comprometidos con la diversidad y la diferencia, a partir de la reflexión sobre su propio quehacer y del efecto de los currículos de formación de profesores en el área de lenguaje en la formación del profesorado de los países participantes y en América Latina y el Caribe. El trabajo se realizó mediante la metodología de comunidad de práctica (Wenger, 2001), con la idea de construir conocimiento consensuado desde los propios actores implicados, en este caso en la formación de profesores de lenguaje. El resultado del trabajo del área es un sistema curricular y didáctico, del cual se destacan en este artículo los referentes curriculares para la formación del profesorado de lenguaje y comunicación. Se presentan particularmente las implicaciones epistemológicas didácticas en lenguaje que conllevaron esta construcción, como una contribución a la investigación en la pedagogía y la didáctica del lenguaje, en el horizonte de la diversidad y la diferencia.

Palabras clave: formación de profesores, referentes curriculares, variantes e invariantes curriculares, diversidad, diferencia, comunidad de práctica, lenguaje y comunicación.

\begin{abstract}
This article presents results of a curricular and didactic research study framed within the objectives of the ALTER-NATIVE project, ALFA III. Results reflect the cooperative work of Latin American and Caribbean teacher educators who were team members of the ALTER-NATIVE language and communication interest.
\end{abstract}

1 Profesora Asociada, de planta, de la Universidad Distrital Francisco José de Caldas, adscrita al Doctorado Interinstitucional en Educación. Correo electrónico: dorainescalderon@gmail.com

2 Profesora Asociada, de planta, de la Universidad Distrital Francisco José de Caldas, adscrita al Doctorado Interinstitucional en Educación. Correo electrónico: stsolerc@udistrital.edu.co 
Based on both self-reflection on one's own teaching practices and the curricular impact on the professional development of language and communication teachers, the project proposes common guidelines for teacher educators based in Latin America and the Caribbean. The project spouses the idea of communities of practice (Wenger, 2001) aiming at constructing knowledge by consensus with the participation of teacher educators involved in the professional development of language and communication teachers. One of the results is the designing of a curricular and didactic system which referents are highlighted in this article. Particularly, the didactic and epistemological implications carried out by this (co)construction are presented. It is argued that this is a contribution for research on language and communication pedagogy and didactics on the horizon of diversity and difference.

Keywords: teacher professional development, curriculum referents, curricular variants and invariants, diversity, difference, community of practice, language and communication.

\section{UNA BREVE INTRODUCCIÓN}

\section{Algo de contexto general}

Latinoamérica es una de las regiones con mayores desigualdades entre los denominados "ricos y pobres", en términos económicos y políticos. Estas, sumadas a la pobreza generalizada, limitan las oportunidades educativas de la mayor parte de la población. Como lo señala el informe de Políticas educativas de atención a la diversidad cultural, de la Unesco (2005):

[...] los niveles de acceso a la educación, escolarización y logros educativos de los sectores pobres, de los indígenas, de la población negra, de los habitantes de las zonas rurales, y en algunos casos, de las mujeres, son consistentemente los más bajos en comparación con los otros grupos sociales ( $p$. 21).

En este contexto, diversos organismos internacionales mostraron su interés por trasformar las prácticas educativas excluyentes y generaron recomendaciones tendientes a eliminarlas. Con la aprobación de la Declaración Universal sobre la Diversidad Cultural, en 2001 en París, y en 2005 de la Convención Internacional sobre la Protección y Promoción de la Diversidad Cultural, la diversidad se constituyó en la clave para la generación de políticas públicas tendientes a recomponer los tejidos sociales profundamente afectados por la intolerancia y la discriminación. Estos documentos señalan que los modelos de desarrollo han conducido a una fractura de la convivencia pacífica que hace imperativa la necesidad de una ética global basada en la aceptación de las diferencias culturales, y tendiente al pluralismo. La educación sería una de las responsables de tal tarea. Su función se orientó a "ayudar a comprender el mundo y a comprender a los demás para comprenderse a sí mismo" (Delors, 1996, p. 33). A partir de entonces, la Unesco tomó como bandera la defensa de la diversidad cultural en sus programas de educación. Se propuso entonces un cambio de las políticas homogeneizantes por aquellas que favorecieran la diversidad.

Sin embargo, las prácticas escolares tradicionales profundamente arraigadas; la escasa formación de los docentes; el constante actualismo basado en la improvisación de las políticas públicas que no terminan de marcar unos derroteros cuando estos son retirados, ajustados, criticados, y la poca participación de los maestros en las políticas, han hecho que dichas iniciativas escasamente pasen del papel a la realidad.

En las aulas aún predomina una racionalidad clásica mecanicista y reduccionista en la que el único conocimiento válido o legítimo es aquel producido en los ámbitos científicos mediante prácticas igualmente legitimadas y justificadas en las lógicas positivistas de la modernidad. 
La diversidad se confundió con el folclor, la conmemoración de fechas o los productos artísticos que se tradujeron en nuevas decoraciones de las aulas o en jornadas de la diversidad, en las que una charla o conferencia basta para cumplir con los requisitos impuestos por las políticas de Estado. Y al estar dirigidas a tipos particulares de poblaciones, personas con las comúnmente denominadas necesidades educativas especiales (NEE), indígenas o afrodescendientes, los profesores no se sienten motivados, pues no logran comprender cómo estas diferencias afectan las dinámicas sociales del aula y el éxito educativo, cada vez más centrado en el logro de competencias académicas concretas, estandarizadas y homogeneizantes.

\section{LA FORMACIÓN DOCENTE EN EL ÁREA DE LENGUAJE}

La docencia del lenguaje y las lenguas, en especial de la lengua castellana, ha sido objeto de profundas reflexiones y en cierta medida ha estado supeditada a los avances de la lingüística como ciencia y a las teorías literarias. En las décadas de 1960 y 1970, teniendo de fondo los planteamientos de la lingüística estructural y generativa, la enseñanza de la lengua se limitó a la exposición de las teorías gramaticales, y sus presupuestos sistemáticos, con claro enfoque descriptivo. A mediados de los años 1980 y durante los años 1990, surgió un primer intento por apartarse de esta tendencia teórica, sistemática. Se planteó un cambio hacia el enfoque semántico-comunicativo, en el que la idea de lenguaje pasó de una orientación exclusiva de lengua como sistema a procesos de significación a través de variados códigos y formas de simbolizar. Se introdujo el concepto de situación que se convirtió en el elemento central de los procesos de significación, en el que además se incluyó a los sujetos y sus manifestaciones históricas, sociales y culturales. Esta nueva tendencia se enfocó en los productos comunicativos, sus usos sociales ubicados en contextos reales de comunicación. Sin embargo, las prácticas educativas poco variaron; si bien se abandonó un poco la enseñanza de la ortografía y la gramática, con fuerte carácter memorístico, prevaleció el carácter instrumental y técnico del desarrollo de las habilidades lectoescriturales y orales.

Durante los años 1990, en Latinoamérica, y por disposiciones de organismos internacionales, se generaron políticas educativas para el mejoramiento de la enseñanza de la lectura y la escritura. Políticas que se convertirían en los denominados lineamientos curriculares y que hicieron eco al discurso de las competencias, puestas en la orientación pedagógica del discurso del desarrollo. De allí que las pretensiones del discurso educativo de finales de la década del ochenta para construir saberes significativos en cada uno de los campos del conocimiento, y en particular del lenguaje, se remplazó por el discurso de las competencias; pues los primeros resultaban menos estables y más difíciles de incluir en los currículos; mientras que las competencias definen atributos concretos de los sujetos en el aspecto económico-laboral, y permiten crear sistemas estandarizados de evaluación, más acordes con la mercantilización de la educación.

A partir de estos lineamientos cada institución formadora de educadores, haciendo uso de la autonomía curricular o siguiendo las políticas de los Estados, ha planteado distintos tipos de propuestas tendientes, en mayor o menor grado, a especificar los procesos pedagógicos relativos a los presupuestos de la docencia del lenguaje. En la actualidad algunos programas se centran aún en la enseñanza de las disciplinas lingüísticas a partir de los niveles de lengua, ampliándolos, eso sí, hasta el nivel del discurso; otros han optado por problematizar los fenómenos del lenguaje de acuerdo con sus dimensiones cognitiva, social, ética, estética o de interacción.

El gran déficit en los programas de formación de profesores de lenguaje sigue siendo, primero, la centralidad de la lengua española, en detrimento de las cientos de lenguas indígenas que persisten en Latinoamérica y de otras lenguas, como 
las de señas de los distintos países del mundo; también se desconocen otros sistemas de comunicación como el braille y aspectos culturales de la enseñanza como: el reconocimiento de diversas formas de expresión, conocimiento, aprendizaje e interacción, provenientes de la diversidad de subjetividades que acceden cada vez más a los contextos escolares en todos los ciclos de formación. Este déficit proviene, en esencia, de una visión limitada del lenguaje en el que predomina el concepto de lengua y en la que, en general, el español se asume como la única lengua oficial de la escuela, a pesar de los variados e importantes intentos por establecer la educación bilingüe, con el español como segunda lengua, en los lugares donde hay población indígena, o de incluir las lenguas de señas y el braille en los contextos escolares.

\section{LA EXPERIENCIA DE ALTER-NATIVA ${ }^{3}$}

Desde el panorama educativo expuesto, el equipo de las tres áreas Alter-Nativa se propuso la tarea de generar lineamientos curriculares para la atención a la diversidad, no con el ánimo de atender a los llamados de las políticas públicas sino con la convicción de que la formación de profesores es un factor determinante de la calidad de la educación, de su pertinencia y de sus posibilidades en la consecución de sociedades más justas y equitativas, y desde la firme convicción de que el cambio es posible solo si se parte de la propia

3 Alter-Nativa: "Referentes curriculares con incorporación tecnológica para facultades de educación en las áreas de lenguaje, matemáticas y ciencias para atender poblaciones en contextos de diversidad" fue una acción subvencionada por la Unión Europea en el marco del Programa América Latina Formación Académica (Alfa III-2) de la Convocatoria 2010 y con vigencia 2011-2013. Alfa es un programa de cooperación internacional entre la Unión Europea y América Latina para la Educación Superior. De este proceso y como uno de los resultados de esta acción, se generó la Red Alter-Nativa constituida durante el desarrollo del proyecto, por el equipo de formadores de profesores, investigadores en educación, ingenieros formadores de ingenieros, de ocho universidades de América Latina, tres de Europa y cuatro entidades cooperantes. Hoy, la Red Alter-Nativa Educación y Tecnología en y para la Diversidad aglutina a los socios iniciales y nuevas instituciones, alrededor de proyectos de investigación y de acción, en torno a la educación accesible para todos. experiencia de los actores implicados en la formación docente: los formadores de educadores, los estudiantes para profesores y los estudiantes de la educación básica y media; y además, con el criterio de que se involucrarán las áreas específicas de formación. En otros términos, no referentes en abstracto, sino sobre su quehacer en las aulas como profesores de un área específica de conocimiento; en este caso, formadores en lenguaje y comunicación.

En ese sentido, la labor de formular referentes curriculares estuvo orientada por tres elementos:

- El reconocimiento de la diversidad lingüística y cultural en América Latina y el Caribe, resultante, fundamentalmente, de la diversidad de los pueblos indígenas originarios y del legado de las culturas africana y española que dieron vida a la rica variedad de expresiones del continente.

- Las orientaciones planteadas por el objetivo macro de Alter-Nativa, Alfa III: proporcionar elementos para la formación de profesores en un contexto que demanda la atención de las poblaciones con necesidades educativas diversas (NED) ${ }^{4}$ e integrando en este proceso el uso significativo de las TIC.

- La identificación de la relación entre la cualificación de la formación docente y la posibilidad de generar "una educación para todos a lo largo de toda la vida" (Delors, 1996; CRES, 2008, citado por Calderón, 2014).

Con estos criterios se constituyó la Comunidad Alter-Nativa de Lenguaje y Educación (CALE), compuesta por las seis universidades socias participantes en el área de lenguaje y tres entidades

4 Esta expresión emerge en el marco del desarrollo del proyecto Alter-Nativa Alfa III, como propuesta de las comunidades de lenguaje, matemáticas y ciencias, para reconocer necesidades educativas en todas las poblaciones sin distingo de tipo de necesidad y en el contexto de la diversidad. 
cooperantes, que son, respectivamente: Universidad Mayor de San Andrés (UMSA), de Bolivia; Universidad Nacional Mayor de San Marcos (UNMSM), de Perú; Universidad Distrital Francisco José de Caldas (UDFJC); Instituto Nacional para Sordos (INSOR), e Instituto Nacional para Ciegos (INCI), de Colombia; Universidad Pedagógica Nacional (UPN), de México; Universidad de las Regiones Autónomas de la Costa Caribe Nicaragüense (URACCAN), de Nicaragua, y Universidad Centroamericana José Simeón Cañas (UCAJSC), de El Salvador. La Figura 1 representa la comunidad CALE.

Este grupo se focalizó en el trabajo con tres grupos poblacionales como beneficiarios de la acción, de acuerdo con la experiencia de las universidades socias y las instituciones cooperantes: pueblos indígenas, poblaciones con limitación sensorial auditiva y poblaciones con limitación sensorial visual. Además, se propuso considerar la formación de profesores en el área de lenguaje para el ciclo de primaria, en particular para la enseñanza del español como primera o segunda lengua. También se consideró como fundamental inscribir la reflexión como educación de poblaciones con $\mathrm{NED}^{5}$, en el entendido de que todos los actores educativos somos diversos, diferentes y requerimos miradas individuales y colectivas por parte del currículo, de la didáctica y del profesor.

\section{Problemáticas de partida para CALE}

La comunidad CALE realizó un rastreo de currículos de sus propios países e instituciones, e identificó características de la formación de profesores en el área de lenguaje y para el ciclo de primaria. Como resultado, identificó algunos aspectos que se constituyeron como fundamentales para la generación de los referentes curriculares:

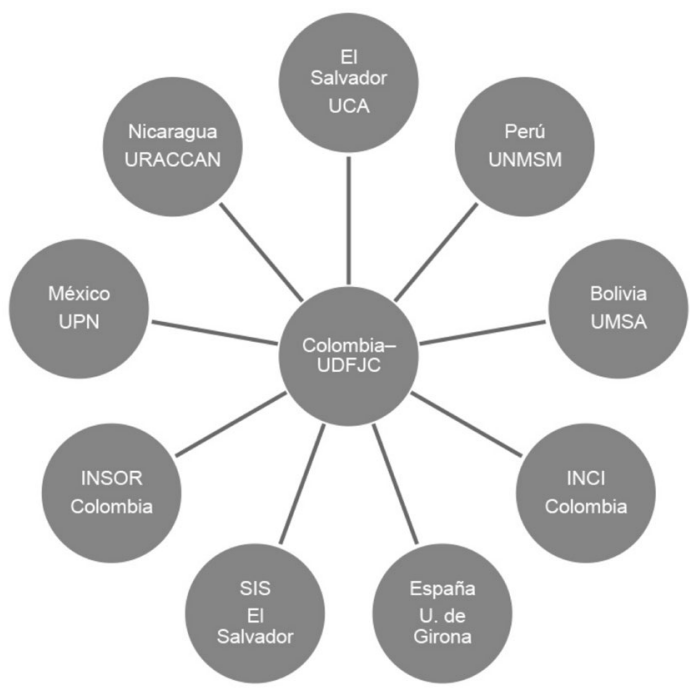

Figura 1. Comunidad CALE

Fuente: elaboración propia.

5 El equipo Alter-Nativa propuso como uno de los acuerdos de todo el proyecto, pasar de la expresión necesidades educativas especiales a necesidades educativas diversas (NED), como un reconocimiento al carácter fenoménico y natural de lo diverso, no solamente cuando existen condiciones acuciantes como las limitaciones sensoriales (sordera o ceguera) cognitivas, físicas, etc., sino la diferencia como una condición natural a la existencia misma del humano y de lo humano. 
- El acceso a la educación en condiciones de vulnerabilidad sensorial o sociocultural.

- La convivencia en diversidad lingüística y cultural.

- El uso de las tecnologías en las aulas de lenguaje.

- La dificultad en la identificación y selección de los contenidos específicos para formar en el área de lenguaje. El reto de formar profesores, en el marco de una educación de alta calidad, como un derecho humano (Pigozzi, 2008).

Y desde estos puntos de partida, CALE propuso los siguientes objetivos:

- La generación de elementos orientadores para la implementación de políticas educativas para la formación de profesores en el área de lenguaje y comunicación (LyC).

- La construcción de elementos orientadores para la construcción de currículos de formación de profesores en el área de LyC.

- La generación de referentes para el diseño de acciones específicas en aulas de LyC con poblaciones diversas: con limitaciones sensoriales (sordera y ceguera), poblaciones indígenas de Latinoamérica y estudiantes en general.

- Presentación de la experiencia de construir, de manera consensuada, referentes curriculares y propuestas didácticas, mediante la metodología de comunidades de práctica.

\section{COMUNIDADES DE PRÁCTICA COMO ELECCIÓN METODOLÓGICA}

Para el desarrollo de los objetivos, CALE asumió la metodología de comunidad de práctica ${ }^{6}$, insistiendo en la necesidad de producir conocimiento consensuado, más allá de la idea del experto y de la intervención externa. Esta metodología, en términos generales, se caracteriza por la

6 También fue la metodología adoptada por las comunidades de ciencias y de matemáticas Alter-Nativa. configuración de una comunidad humana constituida por personas que comparten una preocupación o una pasión por un quehacer y que quieren aprender a hacerlo mejor, de manera colectiva, a medida que interactúan con regularidad en ese dominio; esta dinámica permite el desarrollo de una identidad definida por el actuar de la comunidad (Wenger, 2001).

Trabajar en comunidad de práctica posibilita el intercambio de experiencias, la generación de nuevo conocimiento sobre los campos de estudio y sobre la misma práctica de la comunidad; favorece el trabajo colaborativo entre personas con distintos grados de experticia, desde diferentes o iguales campos de conocimiento; permite crear redes de conocimientos particulares y transversales. En consecuencia, asumir esta forma de trabajo en un espacio que pretende la cualificación de la práctica docente, favorece la construcción de conocimientos en torno a aspectos particulares de dicha práctica, lo cual, a su vez, motiva vivencias de aprendizajes colectivos y colaborativos.

En la comunidad CALE se forjaron prácticas (con sus actores), formas de organización y resultados consensuados, en particular para la formulación de referentes curriculares para la formación de profesores de lenguaje (particularmente orientados a la enseñanza del español o castellano). Como aspectos de la experiencia de comunidad de práctica CALE, se destacan principios asumidos y reflexionados como:

- La conformación de la comunidad de práctica CALE.

- El establecimiento de metas.

- La práctica centrada en la indagación.

- La generación de identidad CALE.

- Los significados elaborados en el sistema didáctico generado.

A continuación se presentan las fases desarrolladas desde esta metodología, atendiendo al objetivo general de construir referentes curriculares (Figura 2): 


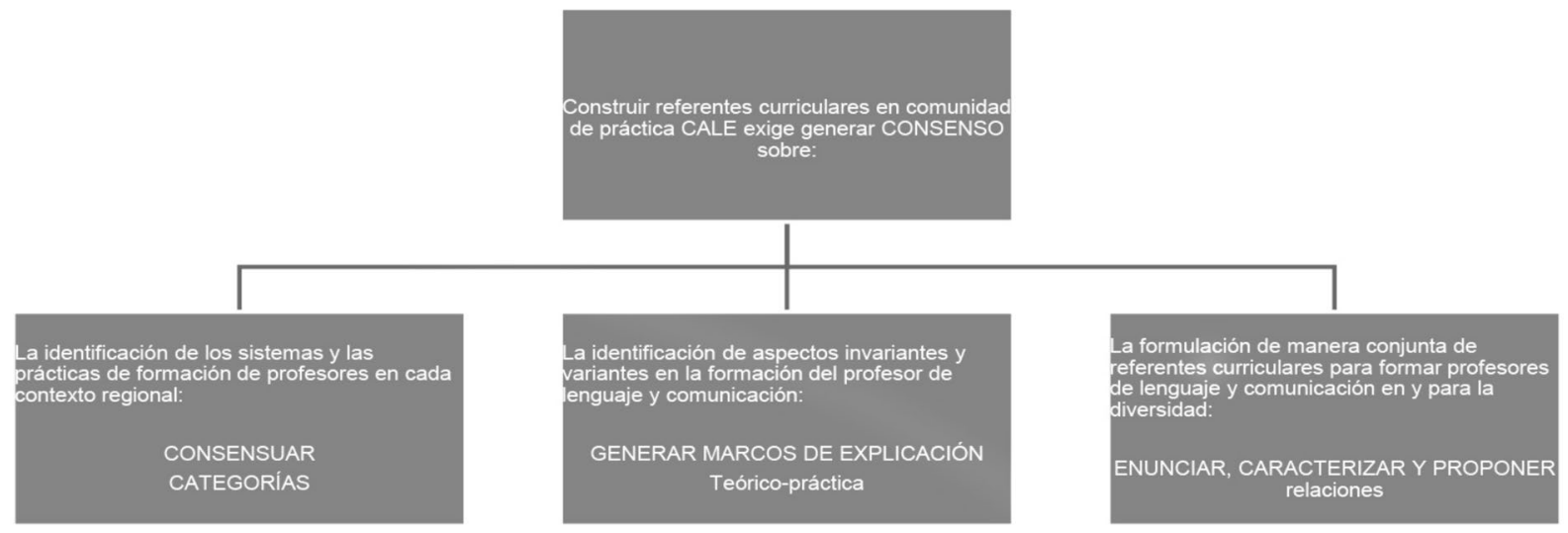

Figura 2. Acciones en la comunidad de práctica CALE

Fuente: Calderón (2014, p. 33).

\section{LOS RESULTADOS DE CALE}

Atendiendo al objetivo, CALE asumió una propuesta basada en la identificación de variantes e invariantes curriculares. Este procedimiento reflexivo facilitó reconocer aspectos fundamentales que hacen posible una educación para todos, cualquiera que sea su condición de diferencia.

\section{La identificación de variantes e invariantes en la formación de profesores en y para la diversidad}

La definición de variantes e invariantes curriculares como base de los referentes para la formación de profesores de lenguaje y comunicación facilitó la identificación de aspectos fundamentales en una educación para todos, y propició la comprensión e interpretación del campo de la educación como campo de saberes y de prácticas y, en él, la determinación de lo invariante (como principio y como necesidad en la formación del profesional educador) y de lo variante (como factor de diversidad), que por principio debe

7 Todo el sistema de productos reposan y están disponibles en la plataforma http://alternativatutor.udistrital.edu.co/inicio Los referentes pueden ser descargados en http://die.udistrital.edu.co/ publicaciones/referentes_curriculares_con_incorporacion_tecnologias_para_formacion_del_profesorado_0 ser respetado y tenido en cuenta en la formación y en la interacción pedagógica (Calderón, 2014, p. 40).

Los invariantes en la formación de profesores se asocian con principios compartidos por las comunidades que presentan un alto de grado de estabilidad $y$, por tanto, son escasamente modificados por los entornos. En términos de lo curricular, se vinculan con aquello que permanece en el currículo, y que se relaciona con los conocimientos, los sujetos y los aprendizajes. Por lo que la búsqueda de invariantes en CALE se orientó hacia estos tres elementos. De esta manera se encontraron los siguientes tipos de invariantes:

- Orientaciones (pronunciamientos, generalmente filosóficos o de carácter político) para la educación y, por ello, para la formación del profesor de lenguaje y comunicación.

- Principios para el desarrollo del lenguaje y la comunicación, aspectos o factores del desarrollo del lenguaje y la comunicación en los sujetos.

- Aspectos constitutivos de los sistemas de los lenguaje (Calderón, 2014, p. 35).

Los variantes curriculares, por el contrario, buscan reconocer componentes particulares, diferenciadores, susceptibles de cambio de acuerdo con los contextos socioculturales y a las características de los 
sujetos. Para el área de lenguaje y comunicación se hallaron los variantes que se detallan a continuación:

- Poblacionales: desde la perspectiva de la diferencia de condiciones sociales, étnicas, sensoriales, culturales, cognitivas, generacionales, etcétera.

- Mediacionales: a partir de los sistemas y de la semiosis: lingüísticas (como lenguajes que median interacciones), comunicativas, instrumentales, mediáticas.

- Lingüísticos, sistémicos y discursivos: se trata de reconocer diferencias en cada sistema lingüístico en cuanto a sus exigencias de conocimiento, uso e incorporación sociocultural.

- Socioculturales: en los procesos y las prácticas particulares en la comunicación y en las expresiones de las culturas y de los grupos sociales. Los distintos contextos de comunicación exigen el reconocimiento de diferentes funciones, intenciones, registros y usos de estrategias de comunicación.

- Políticos: que diferencian sistemas educativos y generan marcos de acción en atención a condiciones diferentes de los sujetos y de los contextos (Calderón, 2014, p. 36).

En la Tabla 1 se detallan los variantes e invariantes para la formación de profesores de lenguaje y comunicación en y para la diversidad.

\section{Las resignificaciones en la didáctica del lenguaje y la comunicación ${ }^{8}$}

A partir del proceso desarrollado por CALE, se considera pertinente hacer explícito el trabajo de resignificación de algunos de los conceptos centrales en

8 Esta propuesta de resignificaciones fue presentada como conferencia magistral en el VI Congreso Internacional de Investigación y Didáctica de la Lengua y la Literatura y VIII Foro Nacional sobre la Enseñanza de la Literatura "Josefina de Ávila Cervantes", Departamento de Letras, Universidad de Sonora, 29, 30 y 31 de octubre de 2014, en Hermosillo, Sonora (México). Conferencia de Dora Inés Calderón: "Formar profesores de lenguaje y comunicación en y para la diversidad: una propuesta desde América Latina y el Caribe". la formación de profesores de lenguaje; en particular cuando esta resignificación emerge como resultado de situar el propósito de construir referentes curriculares en el horizonte de la diversidad y la diferencia y de la tecnología, como factores ético-políticos que definen una educación para todos y con todos. Como resultado del proceso de construcción de referentes curriculares en CALE y de su aplicación didáctica, hemos identificado cuatro lugares de resignificación: epistemológico, comunicativo, éticopolítico e instrumental. A continuación se presenta cada uno de estos lugares y las resignificaciones requeridas para la generación de los referentes curriculares propuestos.

\section{Requerimientos desde lo epistemológico}

- Recuperar una lingüística del lenguaje centrada en las macrofunciones del lenguaje: como facultad, como conocimiento y como interacción. Esta opción epistemológica del lenguaje proporciona elementos para comprender a todos los sujetos (cualquiera sea su condición sensorial, lingüística física o sociocultural) como sujetos de lenguaje y de discurso. Por ello facilita la identificación de lo invariante en la formación en lenguaje tanto del profesor como de cualquier sujeto discursivo, y en lo invariante en el lenguaje como facultad y sistema. De igual manera, permite el reconocimiento e incorporación de lo variante en el desarrollo del lenguaje y en la expresión de los variados sistemas de comunicación.

- Orientar la acción didáctica para definir y delimitar la transposición didáctica del saber que se va a enseñar. Esta resignificación en el campo didáctico permite comprender la diferencia entre el campo de las ciencias del lenguaje que lo explican y este mismo campo situado en el marco de las relaciones curriculares y didácticas. Reflexión que exige objetivar el lenguaje (en todas sus condiciones, expresiones y experiencias) como objeto para ser enseñado y aprendido, en contextos y situaciones particulares. 
Tabla 1. Invariantes y variantes curriculares en CALE

\begin{tabular}{l} 
Invariante curricular \\
\hline $\begin{array}{c}\text { Aspectos básicos para la formación del } \\
\text { profesor de lenguaje }\end{array}$ \\
En el nivel epistemológico del lenguaje \\
El lenguaje es facultad humana. El reconocimiento del lenguaje \\
como una facultad común a todo humano y a todo grupo social y \\
cultural. Se realiza y se manifiesta en experiencia lingüística, discur- \\
siva, comunicativa y semiótica socioculturalmente situada. \\
Las lenguas son sistemas semióticos. Todas las lenguas están estruc- \\
turadas como sistemas simbólicos, producto sociocultural, identita- \\
rio e histórico de toda comunidad; por ello, factor de transmisión y \\
de generación de cultura y de conocimiento. Las lenguas son a la \\
vez el contenedor y el vehículo de una cultura.
\end{tabular}

La diversidad de las funciones socioculturales de las lenguas (jerarquías y códigos en el uso de las lenguas).

Variaciones en la organización lingüística de la experiencia por contextos de diversidad

La diversidad lingüística es parte de la experiencia en la vida social. La diversidad no es una teoría, es un hecho y se manifiesta en experiencias sociolingüísticas de tipo dialectal, bilingüe o plurilingüe y en relaciones lengua materna, lengua primera, L2, L3, etcétera. Todo grupo humano desarrolla otros sistemas semióticos de comunicación distintos al verbal y reproduce en ellos procesos de significación y de comunicación, similares y traducibles a las lenguas naturales.

\section{En el nivel epistemológico de la pedagogía}

La relación entre educación, pedagogía y didáctica (del lenguaje) es constitutiva de los fundamentos de la formación de un profesor (de lenguaje). Esta relación da razón de la acción educativa, del ejercicio del profesor y del contexto de la educación.

El contexto de la educación es un constructo cultural y se constituye por: la existencia de las instituciones educativas, sus principios, criterios organizaciones, disponibilidad y acceso tecnológico y científico, y los agentes formadores (roles y funciones).

La acción educativa se manifiesta como un proyecto pedagógico. Relaciona los principios y las instituciones con las prácticas educativas en los distintos ámbitos, modalidades y niveles de la educación. Tiene un carácter teleológico: todo proyecto educativo se fija metas a largo plazo, articula la dimensión de la formación y de la educación.

El profesor es uno de los principales agentes en el proceso educativo. Su formación requiere de la definición de políticas o directrices para la acción en el área de lenguaje y comunicación.

\section{Variante curricular}

Aspectos diferenciados por contextos, condiciones y poblaciones para la formación del profesor de lenguaje

En el nivel epistemológico del lenguaje

Diversidad de manifestaciones lingüísticas, discursivas, comunicativas y semióticas que expresan las variaciones socioculturales de las comunidades.

Las distintas lenguas y sus gramáticas.

Las lenguas (re)producen distintas cosmovisiones y representaciones.

Las lenguas promueven distintas identidades sociales.

La diversidad lingüística se manifiesta tanto en las variaciones de una lengua como en la variedad de lenguas (orales y de señas).

Las lenguas conviven en la experiencia de los sujetos y en las prácticas socioculturales.

Los grados de dominio de las lenguas.

La diversidad de las funciones socioculturales de las lenguas (jerarquías y códigos en el uso de las lenguas).

Variaciones en la organización lingüística de la experiencia por contextos de diversidad

Los distintos sistemas de comunicación no verbal (pintura, música, imagen, lenguajes formales...) significan y comunican en distintos sistemas de signos.

Las formas paralingüísticas acompañan y complementan la producción de significado en las lenguas, y cooperan con la continuidad de la interacción.

\section{En el nivel epistemológico de la pedagogía}

Variación de sistemas educativos por países, por contextos o por culturas, podrán diferenciarse: políticas, diseños y enfoques curriculares diferenciados, niveles educativos y modalidades de enseñanza.

Distintas propuestas pedagógicas por factores: culturales, contextuales, tendencias pedagógicas, programas diferenciales para la enseñanza de las lenguas.

Prácticas diferenciales de los docentes: características de las prácticas por contextos, rol del docente, rol estudiante, contenidos, objetivos y directrices pedagógicas institucionales. 
Invariante curricular

El ejercicio del profesor de lenguaje manifiesta las relaciones didácticas construidas para llevar a cabo los procesos de enseñanza y de aprendizaje.

Las relaciones didácticas articulan contenidos curriculares del área de lenguaje, estudiantes y profesores, relación que da como resultado el establecimiento de estrategias de trabajo en el aula, el uso de materiales y de mediaciones tecnológicas y la aplicación de sistemas de evaluación.

La acción didáctica del lenguaje en el nivel primario implica el desarrollo de las cuatro acciones discursivas básicas (hablar, escuchar, leer y escribir); la iniciación a la experiencia de la lectura y la escritura; la diversidad textual y discursiva (formación del sujeto discursivo, ético, político, epistémico, lúdico); la experiencia literaria.

\section{Variante curricular}

\section{Diversidad de relaciones didácticas}

Adecuaciones contextualizadas: selección, elaboración y uso de diversos contenidos del área de lenguaje y de objetivos, de acuerdo con características poblacionales, contextuales, pedagógicas y mediacionales.

Distintas formas de evaluación en relación con criterios curriculares, didácticos y contextuales (población y recursos).

Diferentes estrategias de trabajo del docente por grados y modalidades.

Diferencias entre aprendizaje y desarrollo de lenguaje.

Diversidad en el uso y la experiencia en el aula: el rol de las lenguas (lenguaje[s] y enculturación y diálogo intercultural); escrituras y lecturas múltiples; hipertextualidades presentes en las experiencias culturales e interculturales.

Diversidad de manifestaciones literarias: por géneros, por culturas, por formas de expresión (oral, escrita, señada, pictórica, musical, etcétera).

Diversidad de recursos tecnológicos o informáticos por parte de los docentes y los estudiantes.

Fuente: Calderón (2014, pp. 38-40).

\section{- Comprender que el estudiante desarrollará} su capacidad de ser autónomo. Es decir, situar un gran propósito de la formación en lenguaje y comunicación, en cualquier nivel de escolaridad: la formación del sujeto discursivo. Las implicaciones pedagógicas y didácticas de esta resignificación son innegables y sitúan una dimensión ético-política para la pedagogía del lenguaje y la comunicación, que han de orientar las decisiones curriculares y didácticas, los modos de concebir los contenidos, las experiencias y los procesos de formación en esta área.

\section{Requerimientos desde lo comunicativo}

Derivado de las resignificaciones epistemológicas, se hace necesario reconocer que:

- Para el área, el lenguaje tiene un doble lugar como:

\section{- Práctica natural para la comunicación didác-} tica de los contenidos del área. Este aspecto requiere ser comprendido por los profesores, pues las prácticas de lenguaje (el uso de las lenguas y de otros sistemas de comunicación) son naturales a todo grupo humano, independientemente de la reflexión que se realice a cerca de esta práctica sociocultural. Así, en las aulas de lenguaje se comunican los objetos del lenguaje mediante el lenguaje (en este caso privilegiadamente las lenguas). Este se convierte en un lugar para la reflexión del profesor de lenguaje y comunicación: ¿Cómo comunico lo que comunico en mi acto de enseñanza?

- Objeto de conocimiento. En el área de lenguaje y comunicación, el objeto de la enseñanza y del aprendizaje es el mismo lenguaje, la experiencia de lenguajear (Echeverría, 1996). Reconocer los contenidos de este objeto requiere que el profesor comprenda su relación con las prácticas diarias de lenguaje y comunicación.

- La comunicación/visualización (presentación) de los contenidos requiere una mirada semiótica y cultural que implica:

- Integración de tecnologías, entre ellas las TIC. Este aspecto comunicativo se sitúa en la comprensión de la relación entre las multirrepresentaciones presentes en las culturas y los soportes materiales de tales representaciones (tecnologías). En este sentido, potenciar la relación comunicativa para comprender más efectivamente en las aulas, exige integrar distintas tecnologías como aportantes y 
necesarias para responder a dos criterios: el acogimiento de distintas formas de representar por los diferentes grupos socioculturales presentes en las aulas y el desarrollo de experiencias con distintas tecnologías como una forma de cualificar el conocimiento de los objetos enseñados.

- Resignificación de la oralidad, la escucha, la escritura y la lectura hacia la noción de hipertextualidad. La noción de hipertexto constituye una de las expresiones de la incorporación de múltiples formas de producción discursiva y textual contemporánea. Estas opciones discursivas implican reformulaciones semióticas, gramaticales, estéticas y culturales que proponen otras maneras de hablar, de escuchar, de leer y de escribir que el aula debe considerar e incorporar.

- Variación de las modalidades de interacción profesor/estudiante: física, virtual, síncrona, asíncrona. Las tecnologías, en especial las de tipo digital, proponen nuevas formas de interacción, con temporalidades y espacialidades distintas que imprimen otras maneras de relación y el uso de herramientas diferentes para favorecer estas interacciones. Los aprendizajes implicados en estas experiencias deben ser asumidos como apropiación y desarrollo de nuevas prácticas interactivas y su papel en los procesos de enseñanza y de aprendizaje.

\section{Requerimientos desde lo ético-político}

A partir de la diversidad/diferencia como fenómeno natural a la condición de existencia de las comunidades humanas y, por ello, a la educación y sus mediaciones posibles, identificamos dos aspectos involucrados en la didáctica del lenguaje y la comunicación, que exigen ser resignificados:

\section{- La mirada sobre el sujeto/actor de la educa-} ción (profesor, estudiante, comunidad educativa) como sujeto discursivo de la escolaridad y para la participación social. Esta mirada nos lleva a proponer que las acciones discursivas básicas hablar/señar, escuchar, leer y escribir constituyen las que han de centrar los propósitos de la formación en el área de lenguaje y comunicación. Tales acciones se mantienen a lo largo de la vida como experiencias centrales para el sujeto discursivo; por esta razón los currículos del área han de consolidar propuestas para el desarrollo de las cuatro acciones y su relación con la experiencia de lenguaje en todos los ámbitos de la vida.

- Los entornos cibernéticos asumidos de manera crítica, como escenarios configurados y configurantes a partir de múltiples convergencias (económica, tecnológica, cultural...). Comprender el papel sociocultural de las tecnologías digitales y su aporte a la generación y al desarrollo de entornos que favorecen la comunicación inmediata, a acercar lo remoto, a aumentar las posibilidades de información, a proporcionar condiciones para el acceso de personas con limitaciones sensoriales, cognitivas, motoras, etc., es una de las responsabilidades de la educación. También lo es generar condiciones para llevar a cabo y formar la mirada crítica sobre estos entornos, en cuanto productores culturales y configuradores de nuevas subjetividades y de nuevas prácticas de interacción social.

\section{Desde lo instrumental}

Se propone que en la didáctica del lenguaje y la comunicación los aspectos que caracterizan los instrumentos y los soportes materiales de los recursos y de las mediaciones empleadas en las relaciones enseñanza/aprendizaje sean asumidos como mediación instrumental y como mediación semiótica. Este hecho implica tres resignificaciones:

- La comprensión y la inclusión de saberes relacionados con los formatos textuales e hipertextuales y discursivos propios de los entornos digitales y de los dispositivos creados para tal fin. Es un hecho que aprender a leer y a escribir en entornos digitales exige 
tanto comprender los modos de producción discursiva en tales entornos como aprender sobre las tecnologías que facilitan esta producción. Un aprendizaje sobre el uso de los dispositivos y sobre las gramáticas de los entornos cibernéticos.

- El desarrollo de prácticas para el acceso, la utilización y la producción de textos e hipertextos y para la comprensión de las dinámicas de navegación en la web. El profesor requiere comprender que un tipo de prácticas necesarias, que exige aprendizajes particulares es la práctica de navegación en la web. Su desarrollo exige condiciones específicas de los entornos del aula, disponibilidad de equipos y de conectividad e incorporación de espacios escolares para estos desarrollos.

- El acceso a campos léxicos provenientes de los entornos virtuales, y su apropiación. Este es un aspecto fundamental para el campo del lenguaje y la comunicación, pues representa el desarrollo de las mismas lenguas y la generación o ampliación de los campos semánticos y léxicos, que requieren ser apropiados, reflexionados y cualificados.

La formulación de los tres tipos de referentes curriculares $^{9}$

Como producto de la reflexión estructural de los que pueden ser los aspectos fundamentales en la formación de profesores de lenguaje y comunicación,

9 En la construcción de los referentes curriculares para la formación de docentes de lenguaje en y para la diversidad participaron los siguientes profesores-investigadores: Dora Inés Calderón, Sandra Soler Castillo, Mirian Glidis Borja Orozco, Germán Muñoz González, Gloria Rojas Álvarez y Giovanna Patricia Medina, de la Universidad Distrital Francisco José de Caldas, Colombia; Ernesto Díaz Couder Cabral, de la Universidad Pedagógica Nacional, México; Consuelo Lizeth Blandón Jirón, Blanca Nevai Centeno Bravo, Obed Zeledón Membreño, de la Universidad de las Regiones Autónomas de la Costa Caribe Nicaragüense, Nicaragua; María Isabel Ginocchio Laínez Losada, Esther Espinoza Reátegui, César Ernesto Gómez, Rolando Rocha Martínez, de la Universidad Nacional Mayor de San Marcos, Perú; Ada Janeth Zarceño y Patricia Carolina Andreu, de la Universidad Centroamericana José Simeón Cañas, El Salvador; Virginia Ketty Arce y Tania Sáenz Cortés de la Universidad Mayor de San Andrés, Bolivia; Lilly Portilla Aguirre del Instituto Nacional para Sordos (INSOR), Colombia.
CALE propone tres tipos de referentes, en atención a la comprensión de distintos factores que van a constituir la formación profesional integral de un profesor de esta área. Por esta razón se proponen tres tipos de referentes curriculares: i) para la formación profesional ético-política, ii) para la formación profesional pedagógica como profesor de lenguaje y la comunicación, y iii) para la formación profesional didáctica en lenguaje y comunicación.

Los referentes curriculares (RC) se construyen desde la identificación de los variantes e invariantes curriculares ya mencionados, y recogen lo que, a juicio de CALE, habría de estar presente en la formación de profesores de lenguaje y comunicación. Se consideran como marcos de comprensión de aspectos necesarios y esperados en la formación y en la acción del profesorado y en los saberes que han de configurar los sistemas curriculares en el campo del lenguaje y la comunicación. Constituyen la base referencial y el factor de articulación para y entre propuestas didácticas, como en el caso de CALE, para la producción de guías de integración TIC y de objetos virtuales de aprendizaje (OVA). Por ello proporcionan criterios orientadores actualizados y desafiantes para la educación -en este caso en lenguaje y comunicación-. Como características de estos RC encontramos:

- Abiertos a los cambios y a la diversidad de contextos sociales y culturales. Por ello, flexibles (proporcionan apertura curricular).

- Orientadores de las acciones curriculares en las instituciones educativas.

- Propenden por el reconocimiento del otro y de lo otro por la acción con los otros.

- Facilitan la comprensión de quién es el sujeto por formar.

- Mantienen la unidad dentro de la diversidad de situaciones y contextos (Calderón, 2014, p. 41).

En la Tabla 2 se presentan los tres tipos de referentes curriculares construidos ${ }^{10}$ :

10 Se presenta aquí la tabla síntesis de todos los referentes construidos. No obstante, se recomienda la lectura del libro completo de Calderón (2014). 
Tabla 2. Referentes curriculares para la formación del profesorado de lenguaje y comunicación en y para la diversidad

\section{Referentes tipo 1: para la formación profesional ético-política del profesional pedagogica del profesor profesor}

Referente 1. La formación del profesorado en y para la diversidad requiere concebir al maestro como profesional de la educación.

Referente 2. La formación del profesorado en contextos de diversidad exige pensar la articulación de principios, políticas y acciones de todo el sistema educativo.

Referente 3. La formación del profesorado precisa enfatizar que la educación es para todos, cualquiera sea su condición y en función de sus particularidades.

Referente 4. El profesor requiere incorporar y valorar permanentemente, de manera reflexiva y crítica, la experiencia de diversidad en sus prácticas docentes y en todos los ámbitos de la interacción educativa.

Referente 5. La formación del profesorado debe establecer el bilingüismo y el plurilingüismo de las poblaciones diversas como un derecho a las identidades individuales y culturales.

Referente 6. La formación docente demanda desarrollar procesos reflexivos de evaluación equitativos que atiendan las particularidades de poblaciones en contextos de diversidad.
Referente 7. La formación del profesorado del área de lenguaje y comunicación para el ciclo de primaria requiere situar al estudiante desde sus particulares condiciones (sensorial, intelectual, cultural, lingüística, social).

Referente 8. La formación del profesorado supone abordar el lenguaje como un hecho complejo: como la facultad que nos unifica en tanto especie, pero nos diferencia en las posibilidades de representación, y en cuanto seres socioculturales.

Referente 9. Las acciones que posibiliten el reconocimiento y la experiencia con la diversidad de representaciones generadas desde múltiples lenguajes son condición y garantía de coexistencia, respeto e inclusión de comunidades diversas.

Referente 10. La formación docente en una cultura técnica contribuye a la comprensión del papel de las mediaciones tecnológicas en el desarrollo de lenguajes e interacciones para la inclusión.

Referente 11. La formación pedagógica del profesor en el marco de las prácticas colaborativas, contribuye a comprender, cualificar e incorporar las prácticas sociales y culturales de las comunidades diversas, y con ello, a construir una comunidad educativa inclusiva, equitativa y justa.

Referente 12. Las relaciones didácticas construidas desde una perspectiva investigativa posibilitan al docente en formación descubrir saberes sobre la enseñanza y el aprendizaje del lenguaje y la comunicación, orientados a contextos diversos.

Referente 13. Los ambientes educativos que promuevan aprendizajes significativos en lenguaje y comunicación configuran una vía de inclusión de las poblaciones diversas a la escolaridad.
Referente 14. La formación del profesorado de lenguaje y comunicación ha de permitirle comprender que hablar, escuchar, leer y escribir son las acciones discursivas básicas para la interacción sociocultural y para la actividad intelectiva, para el ciclo de primaria.

Referente 15. La creación de situaciones que pongan en práctica la naturaleza semiótica e interactiva del lenguaje sitúa a las personas en condiciones de aprender, interactuar y construir(se) como agentes socioculturales.

Referente 16. La experiencia educativa que promueve la comprensión y producción de textos e hipertextos en variadas situaciones expresivas, y en contextos bilingües, plurilingües e hipermediales favorece el diálogo intercultural escolar.

Referente 17. Las mediaciones tecnológicas orientadas al desarrollo de la lecturabilidad y de la escriturabilidad, potencian la autonomía expresiva de poblaciones diversas, el trabajo colaborativo y los aprendizajes colectivos.

Referente 18. La experiencia lúdica y estética con el uso del lenguaje y los lenguajes impulsa la expresión creativa del sentir y el pensar humanos en contextos de diversidad.

Referente 19. La evaluación, como acción didáctica, es escenario y fuente para la comprensión del efecto formativo que supone el desarrollo de procesos de aprendizaje en contextos de diversidad.

Fuente: Calderón (2014, pp. 42-44). 
Las decisiones epistemológicas en la didáctica del lenguaje y la comunicación

Un gran hallazgo del proyecto lo constituyó la relación entre referentes curriculares y decisiones didácticas a partir de su implementación. Se destacan dos grandes decisiones que tienen implicaciones en la acción didáctica de los formadores de profesores de lenguaje y comunicación:

- Para la Comunidad Alter-Nativa de Lenguaje y Educación, una decisión de tipo epistemológica y metodológica en la relación educación/ diversidad y tecnología es situar la formación didáctica como centro de la construcción de guías para la integración TIC y OVA como apoyo en la didáctica del área. Para ello, se proponen cuatro grandes temas en la formación del profesor de lenguaje y comunicación:
- La experiencia de hablar/señar, escuchar, leer y escribir.

- La diversidad textual y discursiva.

- La experiencia estética y literaria.

- Las mediaciones tecnológicas, lenguaje y diversidad.

En la Figura 3 se ilustran estas relaciones que componen el campo de la formación de profesores de lenguaje y comunicación.

El reconocimiento y la identificación de elementos prioritarios en la formación transversal de los profesores de lenguaje y comunicación y el papel de las TIC.

- Diversidad: poblacional, situacional, mediacional, lingüística, social y cultural...

- Tecnología y mediaciones sociolingüísticas y culturales en educación.

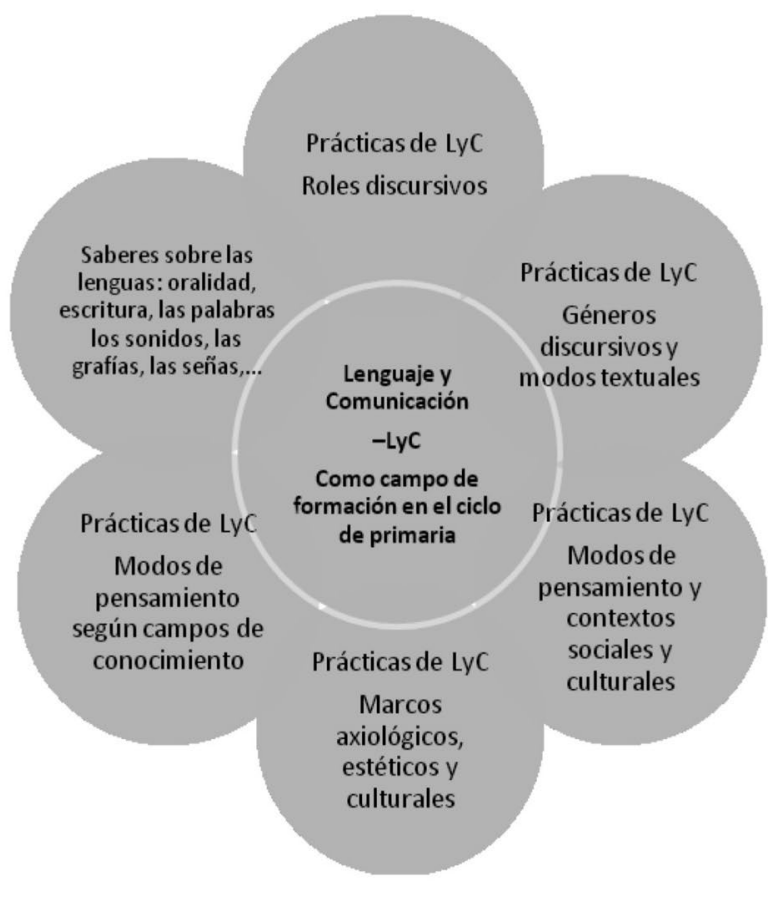

Figura 3. Relaciones que componen el campo de la formación de profesores de lenguaje y comunicación Fuente: Calderón (2014, p. 75). 
- La formación de profesores: a partir de una didáctica de la didáctica del lenguaje y la comunicación. Esto, por cuanto se requiere considerar que el campo de la formación de profesores exige la puesta en escena de una didáctica mediante la cual estos se puedan formar en didáctica del lenguaje y la comunicación. Este ejercicio teórico es exigente, ya que requiere el desarrollo de condiciones metadidácticas para la formación de profesores.

- El entorno ATutor como escenario de y para la formación de profesores, desde una perspectiva de la accesibilidad. Esto, por considerarse uno de los sistemas de gestión de aprendizaje, o learning management system (LMS) más accesibles como gestores de contenidos, en este caso didácticos.

\section{CONSIDERACIONES FINALES}

En la actualidad, hablar de formación de profesores nos lleva a un amplísimo y complejo campo atravesado por intereses políticos y económicos, y por lo que parecieran ser políticas de desprofesionalización, con un proceso de desposesión simbólica del docente, como señala Perrenoud (1996, citado en Torres, 1998), que pone en crisis profunda la retórica de la formación docente.

Compartimos la idea de Rosa María Torres (1998) -reconocida investigadora y teórica sobre formación docente en América Latina-, cuando señala que la formación docente debe entenderse, en la actualidad, en cada condición concreta y partiendo de certezas situadas y de acuerdo con el papel que se asigne a la educación y al sistema escolar:

No hay respuesta única, ni posibilidad de recomendaciones universales, para la pregunta acerca de qué hacer con la cuestión docente y con la formación docente, de manera específica. No la hay por varias razones: la propia gran diversidad de contextos y situaciones entre regiones, países y dentro de un mismo país, que hace difícil (o, en todo caso, irrelevante) la generalización y a la vez separación usual entre "países en desarrollo" y "países desarrollados"; la complejidad y la situación sumamente crítica a la que ha llegado en el mundo el "problema docente", del cual la formación profesional es apenas un aspecto [...] y, en consonancia con todo esto, la imprecisión y los sentidos diversos y en disputa de eso que, vagamente, se avizora hoy como la educación deseada, la "nueva" educación, "la educación del siglo XXI". (Torres, 1998, s.p.).

No es posible entonces hablar de una formación de profesores generalizada; no es lo que se propone en estas páginas ni alimenta el espíritu del proyecto Alter-Nativa. La idea ha sido, en todo caso, construir desde la experiencia situada de los maestros de lenguaje algunos consensos que sirvan de orientaciones para enfrentarnos a esa realidad variable y variante de las aulas de clase. Para entender la escuela y el aula como un espacio de comunicación en el que experimentamos la posibilidad del conocimiento, pero también la posibilidad de ser y construirnos en relación con los otros. En una dinámica dialógica que supone un yo y otro. Un yo y otro diferentes.

En ese sentido, CALE aboga por una educación para la diversidad y la diferencia en la que se entienden como conceptos que se oponen a la igualdad, a la mismidad, a lo idéntico; que fundan su significado en la posibilidad de la descentralidad, en el quiebre, el acontecimiento que irrumpe y abre camino a la experiencia. Si diversidad supone dos puntos, yo y otro; no constituyen ese yo y ese otro la diversidad o diferencia, en cuanto sujetos. La diversidad y diferencia se construyen en ese trayecto que va del uno al otro y viceversa. La metáfora del puente parecería ilustrativa en ese sentido. El puente es el espacio que permite transitar entre dos extremos. Que de alguna manera invita a ese paso. Que se ofrece como destino, como posibilidad de encuentro. Está allí para ser usado, para producir intercambios, para transitar. Para unir, más que para separar. 
Así, desde Alter-Nativa se asume una postura política, en la medida que entendemos que el problema no son las diferencias sino las desigualdades, que como señala Giroux: hacen de la diferencia una etiqueta de conflicto (citado por López, 2002, p. 125). Proponemos que una educación basada en la diversidad tiene como objetivo reflexionar pedagógica y didácticamente para generar propuestas educativas que se caractericen por el acogimiento de las diversidades y de las diferencias, no solo étnicas, sino sociales, culturales y personales. Una educación en y para la diversidad y la diferencia se orienta a toda la comunidad educativa y propone permear por completo el currículo escolar.

\section{RECONOCIMIENTOS}

El artículo es resultado del proyecto "Referentes curriculares con incorporación tecnológica para facultades de educación en las áreas de lenguaje, matemáticas y ciencias, para atender poblaciones en contextos de diversidad". Universidad Distrital Francisco José de Caldas y la Red ALTER-NATIVA. Convocatoria ALFA III, 2010. Unión Europea.

\section{REFERENCIAS}

Calderón, D.I. (ed.) (2014). Referentes curriculares con incorporación de tecnologías para la formación del profesorado de lenguaje y comunicación en y para la diversidad. Bogotá: Universidad Distrital Francisco José de Caldas. Recuperado de: http://die.udistrital.edu.co/publicaciones/referentes_curriculares_con_incorporacion_tecnologias_ para_formacion_del_profesorado_0

Delors, J. (1996). La educación encierra un tesoro (compendio) (p. 44). París, Francia: Unesco de la Comisión Internacional sobre la Educación para el Siglo XXI. Recuperado de: http://www.unesco. org/education/pdf/DELORS_S.PDF

Echeverría, R. (1996). Ontología del lenguaje. Santiago de Chile: Dolmen.
López, M. C. (2002). Diversidad sociocultural y formación de profesores. Bilbao: Ediciones mensajero.

Oficina Regional de Educación de la Unesco para América Latina y el Caribe (OREALC) (2001). Balance de los 20 años del Proyecto Principal de Educación en América Latina y el Caribe (Balance) (p. 158). Santiago de Chile. Recuperado de: http://unesdoc.unesco.org/images/0013/001354/135468s.pdf

Oficina Regional de Educación de la Unesco para América Latina y el Caribe (OREALC) (2005). Segundo estudio regional comparativo y explicativo (SERCE). Análisis curricular. Bogotá, Santiago.

Pigozzi, M.J. (2008). Towards an index of quality education. Paper prepared for the International Working Group on Education. Recuperado de: www. iiep.unesco.org/2008/pigozzi_IWGE_GlenCoveJune2008.pdf

Torres del Castillo, R.M. (1998). Nuevo papel docente. ¿Qué modelo de formación y para qué modelo educativo? Perfiles Educativos, 82 Recuperado de http://www.redalyc.org/articulo.oa?id=13208202

United Nations Educational, Scientific and Cultural Organization (Unesco) (1990). Declaración mundial sobre la educación para todos y Marco de acción para satisfacer las necesidades básicas de aprendizaje. Jomtien. Recuperado de: http:// www.unesco.org/education/pdf/JOMTIE_S.PDF

United Nations Educational, Scientific and Cultural Organization (Unesco) (2001). Declaración Universal de la Unesco sobre la diversidad cultural. Johannesburgo. Recuperadode: http://portal.unesco.org/es/ev.php-URL_ID $=13179 \& U R L \_D O=-$ DO_TOPIC\&URL_SECTION=201.html

United Nations Educational, Scientific and Cultural Organization (Unesco) (2008). Un enfoque de la Educación para Todos basado en los derechos humanos. París, Francia: Fondo de las Naciones Unidas para la Infancia.

Wenger, E. (2001). Comunidades de práctica. Aprendizaje, significado e identidad. Buenos Aires: Paidós. 